\title{
Vers une optimisation de la tenue à la fatigue de pièces forgées intégrant l'histoire mécanique des matériaux
}

\author{
Pierre-Olivier Bouchard, Nicolas Caillet, Yvan Chastel \\ Centre de Mise en Forme des Matériaux (CEMEF) \\ Ecole Nationale Supérieure des Mines de Paris \\ B.P. 207 \\ 06904 Sophia-Antipolis Cedex \\ pierre-olivier.bouchard@ensmp.fr
}

\begin{abstract}
RÉSUMÉ. Les pièces métalliques forgées présentent un fibrage qui leur confère des propriétés mécaniques anisotropes. L'objectif de notre étude consiste à définir la notion de fibrage dans le cadre de la simulation numérique, et à l'utiliser dans l'estimation de la durée de vie de pièces en fatigue. Nous nous focaliserons ici sur la fatigue à haut nombre de cycles, et nous traiterons les aciers contenant des inclusions malléables supérieures à une taille caractéristique. Un nouveau critère de fatigue multiaxial est proposé, sur la base du critère de Murakami, et a été implémenté dans le logiciel éléments finis FORGE3®. Une variable représentant le fibrage est calculée lors de la simulation de forgeage, puis utilisée lors du calcul de fatigue anisotrope. Les contraintes résiduelles obtenues en fin de forgeage sont elles aussi prises en compte dans notre modèle. Enfin une chaîne de simulation virtuelle, intégrant les simulations de forgeage et de fatigue, est réalisée. Les résultats obtenus montrent que l'on peut améliorer la tenue à la fatigue des pièces forgées en modifiant les conditions de mise en forme, telles que le fibrage initial ou encore la géométrie de la pièce. ABSTRACT. Forging of metal components induces grain flow orientation, which gives rise to anisotropic mechanical properties. Our study deals with the definition and the use of this grain flow anisotropy into fatigue life calculation. We have focused on high cycle fatigue, and we deal with steels containing malleable inclusions above a critical size. A new multiaxial criterion has been developed on the basis of the Murakami equation. This criterion has been implemented in the finite element code Forge $3 \circledR$. This computational tool uses a new variable to represent the grain flow orientation provided by forging simulation to account for subsequent fatigue anisotropic behaviour. Moreover, residual stresses resulting from forging are also taken into account to perform the fatigue calculation. Finally, a virtual simulation chain, including Forging and Fatigue calculation is carried out. The final goal is to improve fatigue properties by modifying forging conditions.
\end{abstract}

MOTS-CLÉS : fatigue multiaxiale, fatigue à haut nombre de cycles, forgeage, fibrage, contraintes résiduelles

KEYWORDS: Multiaxial fatigue, High cycle fatigue, Forging, Anisotropy, Residual stresses 


\section{Introduction}

Le dimensionnement constitue une étape importante dans la conception globale de pièces industrielles. Les logiciels éléments finis actuels permettent d'étudier avec précision la tenue mécanique d'une pièce sous différentes sollicitations (statique, dynamique, fatigue, ....). Une fois que le design d'une pièce a été validé par le «service dimensionnement», on demande généralement au «service mise en forme » de définir les procédés, et les gammes permettant d'obtenir la géométrie de la pièce désirée sans endommagement significatif et présentant les caractéristiques mécaniques spécifiées dans le cahier des charges. Cette stratégie présuppose que l'état thermomécanique de la pièce après mise en forme ne modifie pas significativement sa résistance mécanique aux sollicitations initialement étudiées en dimensionnement. Dans certains cas, cette hypothèse peut conduire à des ruptures prématurées des pièces industrielles lors de leur utilisation en service.

De nombreux efforts sont menés actuellement de façon à relier les simulations de mise en forme avec l'étape de conception et de dimensionnement. Ceci nécessite :

- une simulation numérique précise des différentes étapes de mise en forme nécessaires pour obtenir la géométrie de la pièce ainsi que son état thermomécanique final ;

- le transfert des champs mécaniques (ou même microstructuraux) pouvant jouer un rôle important sur la tenue mécanique de la pièce en service ;

- la capacité du modèle de tenue mécanique de pouvoir intégrer cette information en données d'entrée ;

- la capacité du logiciel de dimensionnement de pouvoir intégrer ces champs mécaniques (ou microstructuraux) comme données d'entrée.

L'étude proposée ici consiste à aborder ces différents points de façon à relier forgeage et tenue en fatigue.

Le forgeage d'une pièce métallique induit un fibrage, qui conduit à des propriétés mécaniques anisotropes. Le fibrage correspond à une orientation privilégiée des grains ou des inclusions (ou chapelets d'inclusions) dans un métal ayant subit une opération de corroyage (forgeage, laminage, ...). Le terme fibrage est utilisé par analogie avec des matériaux qui présentent réellement cette caractéristique tels que le bois ou encore certains composites. Dans le cas des métaux il fait référence à l'orientation globale que prend la structure métallurgique en fonction du sens d'écoulement du matériau durant sa déformation

Outre la géométrie finale de la pièce, les logiciels de mise en forme actuels, sont capables de fournir non seulement de simples champs mécaniques (contraintes résiduelles ou déformations plastiques par exemple), mais également des données microstructurales, telles que le fibrage ou la taille de grains. C'est bien cette microstructure qui fait que la pièce de forge a une meilleure tenue mécanique qu'une 
pièce de fonderie ou une pièce usinée. En pratique, les services de conception essaient d'utiliser cette spécificité dans le dimensionnement, mais il n'y a malheureusement pas de lien quantitativement établit entre le fibrage et les propriétés mécaniques de la pièce.

Les calculs de fatigue son très souvent réalisés sur des pièces sans prendre en compte leur histoire mécanique. Le modèle de Dang Van (Dang Van et al., 1989), est souvent utilisé pour prédire la contrainte critique avant rupture d'une pièce en fatigue à haut nombre de cycles (fatigue illimitée). L'équation de Murakami (Murakami et al., 1983) peut également être utilisée en fatigue à haut nombre de cycles, lorsque les défauts atteignent une taille critique. Certaines études (Bouchard et al., 2006) commencent à intégrer quelques paramètres mécaniques significatifs (contraintes résiduelles principalement) dans leurs études de dimensionnement, cependant un travail important doit encore être fait de façon à augmenter le nombre de paramètres pris en compte et à rendre cette approche systématique.

L'objectif de cet article est de montrer comment une étude de fatigue peut être réalisée sur une pièce forgée en intégrant les contraintes résiduelles ainsi que l'anisotropie (due au fibrage) induites par le procédé de forgeage. Le modèle de Murakami, prenant en compte la géométrie des défauts, sera utilisé et étendu de façon à pouvoir l'utiliser pour des chargements multiaxiaux. Une simulation globale, intégrant forgeage et tenue à la fatigue, sera alors effectuée sur un poussoir de soupape. Des modifications de la géométrie finale du poussoir ou encore du sens de fibrage initial seront effectuées de manière à évaluer leur influence sur les valeurs du critère de Murakami. Cette chaîne de simulations pourra être intégrée dans une boucle d'optimisation qui permettra, à terme, d'améliorer la durée de vie des pièces forgées en modifiant certains paramètres de l'opération de forgeage.

\section{Modélisation numérique du forgeage avec calcul du fibrage}

\subsection{Le logiciel FORGE3®}

FORGE3® est un logiciel éléments finis 3D dédié à la mise en forme des matériaux. Il permet de modéliser des matériaux à comportement élastoplastiques, ou élasto-viscoplastiques en grande déformations. FORGE3® est basé sur une formulation mixte vitesse-pression, et utilise des éléments tétraédriques linéaires avec un terme bulle: éléments $\mathrm{P} 1+/ \mathrm{P} 1$. Un remailleur automatique $3 \mathrm{D}$, basé sur l'amélioration topologique des éléments (Coupez et al., 2000), permet de conserver un maillage de bonne qualité lors de grandes déformations. Les non-linéarités sont traitées à l'aide de l'algorithme de Newton-Raphson. 


\subsection{Calcul du fibrage}

La source du comportement anisotrope des pièces forgées en fatigue réside dans le fibrage présent dans les pièces. On peut voir sur la Figure 1 le fibrage d'une pièce corroyée, révélé par attaque macrographique. Ce fibrage est le résultat de l'histoire thermomécanique du matériau et il reflète l'allongement des microstructures et des inclusions dans la direction de la déformation.

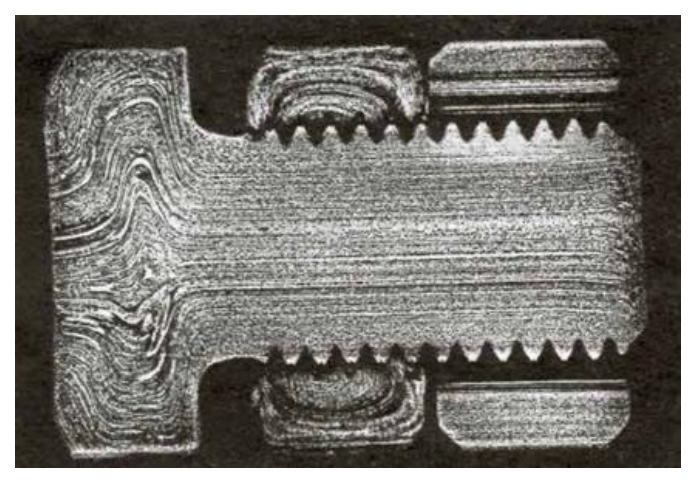

Figure 1: Fibrage d'une pièce corroyée

La caractérisation et la quantification de ce fibrage sont donc des aspects essentiels pour cette étude.

\subsubsection{Principe de la modélisation du fibrage}

Pour le critère de Murakami, utilisé par la suite, la donnée fondamentale à prendre en compte est l'allongement des inclusions. L'idée consiste à représenter la transformation d'une sphère en un ellipsoïde en modélisant les directions principales suivant lesquelles un élément de matière s'est déformé. Pour cela, le tenseur des déformations $F$ est calculé à partir du gradient lagrangien du déplacement :

$$
F_{i, j}=\delta_{i, j}+\frac{\partial u_{i}}{\partial x_{j}^{0}}
$$

Ce tenseur peut être décomposé en une partie sphérique et une partie orthogonale $F=R \cdot U=V \cdot R$ (décomposition polaire). $R$ représente la partie sphérique (mouvement de corps rigide) et $U$ et $V$ représentent la partie orthogonale (appelés respectivement distorsion à droite et distorsion à gauche). Les valeurs propres et les 
directions propres de $\mathrm{V}$ permettent ainsi de déterminer la direction et les dimensions des axes de l'ellipsoïde qui représente une inclusion déformée.

\subsubsection{Application dans le logiciel FORGE3®}

\section{- Introduction d'un fibrage initial}

La plupart du temps, le lopin à partir duquel la pièce est forgée, possède un fibrage. En effet, le lopin a pu subir des opérations telles le laminage ou le filage, qui lui confèrent ce fibrage. Il est donc possible d'introduire une valeur de corroyage initial (homogène sur le lopin) sous forme d'un vecteur. Plus généralement, ce fibrage pourrait être représenté sous forme tensorielle. Cela revient à supposer qu'il existe une direction de déformation privilégiée et deux autres directions équivalentes. Cela représente bien le cas d'une barre par exemple. Ce vecteur est ensuite utilisé pour calculer le tenseur des déformations correspondant. Dans le repère lié au fibrage, pour un corroyage de $c$, le tenseur des déformations $F$ s'écrit :

$$
F=\left[\begin{array}{ccc}
c & 0 & 0 \\
0 & \sqrt{1 / c} & 0 \\
0 & 0 & \sqrt{1 / c}
\end{array}\right]
$$

où $c$ correspond à l'élongation de l'axe principal de l'ellipse de révolution. Il suffit ensuite de repasser dans le repère principal.

\section{- Calcul du fibrage}

Voici les étapes du calcul du vecteur fibrage :

(1) Calcul du tenseur des déformations initial, connaissant le corroyage $F_{\text {initial }}$

(2) calcul du tenseur des déformations relatif au forgeage $F_{\text {forgeage }}$

(3) calcul du tenseur des déformation total $F_{\text {total }}=F_{\text {forgeage }} \cdot F_{\text {initial }}$

(4) calcul de $V^{2}=F \cdot F^{T}$

(5) calcul des valeurs propres et des vecteurs propres de $V^{2}$

(6) vecteurs propres $\rightarrow$ direction des axes de l'ellipsoïde valeurs propres $\rightarrow \frac{\text { longueurdel'axe } i}{\text { rayondela sphèreinitiale }}=\sqrt{\lambda_{i}}$ 
En modélisant le fibrage comme la déformation d'une sphère en un ellipsoïde de révolution, il est possible de le représenter sous forme d'un vecteur. En effet, la conservation du volume impose $\lambda_{1} \cdot \lambda_{2} \cdot \lambda_{3}=1$. De plus, nous faisons l'hypothèse ici que deux des axes sont équivalents. Donc, la connaissance de la direction et de la dimension de l'axe de révolution est suffisante pour décrire le fibrage.

Dans certaines situations, le fibrage pourra avoir une valeur très élevée, et bien évidemment, les inclusions ne se déformeront pas autant, même pour les plus malléables. Une borne est donc imposée au fibrage. Un travail complémentaire devra être réalisé de façon à prendre en compte l'évolution des inclusions (rupture fragmentation) pour de telles déformations.

\subsubsection{Application à l'écrasement d'un lopin}

Dans l'exemple ci-dessous, un lopin est écrasé entre deux outils plats. Le lopin de départ provient d'un barreau et peut ainsi posséder un fibrage initial. La Figure 2 montre l'influence de la prise en compte d'un fibrage initial sur la valeur du tenseur des déformations calculé. Le fibrage initial est obtenu en considérant que le barreau possède un corroyage de 6 , suivant l'axe z. On voit dans ce cas que le fibrage initial est parfaitement pris en compte et qu'il ne modifie pas l'allure de la carte de la composante $\mathrm{zz}$ des déformations.

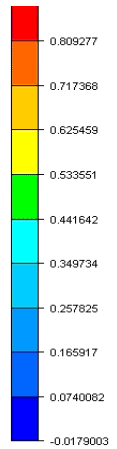

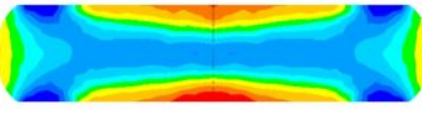

(a) sans fibrage initial

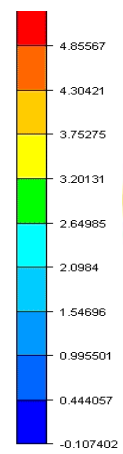

(b) avec fibrage initial

Figure 2: Comparaison des valeurs de la composante zz du tenseur des déformations $F$ entre un lopin avec ou sans fibrage initial

Une comparaison qualitative du fibrage obtenu numériquement et d'un fibrage expérimental est présentée en Figure 3. Les résultats numériques tiennent compte d'un fibrage initial existant dans le barreau (pris arbitrairement équivalent à un corroyage de 6). La Figure 3 montre la composante suivant $\mathrm{z}$ du vecteur fibrage tel qu'elle a été définie précédemment. Cette composante est la plus représentative dans le cas ou le barreau est corroyé dans la direction $\mathrm{z}$. 


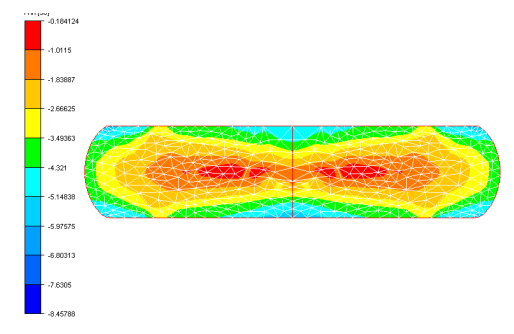

(a) Simulation numérique : composante $\mathrm{z}$ du vecteur fibrage

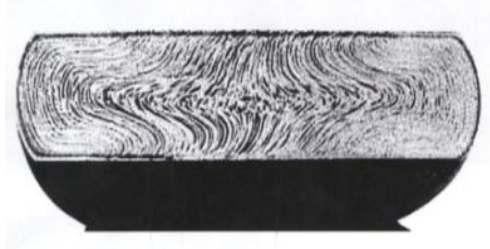

(b) micrographie

Figure 3: Comparaison qualitative entre le fibrage observé expérimentalement et le fibrage calculé avec Forge3 ${ }^{\circledR}$, sur un lopin écrasé

Les valeurs les plus élevées (en valeur absolue) de la composante $\mathrm{z}$ du vecteur fibrage correspondent aux zones dans lesquelles on peut voir un fibrage vertical bien marqué sur la coupe micrographique. En revanche, au centre, on ne distingue plus de fibre sur la coupe micrographique. Les résultats de la simulation montrent bien eux aussi que les valeurs de la composante $\mathrm{z}$ du fibrage les plus faibles sont calculées sur cette même zone.

\section{Calcul de tenue en fatigue intégrant l'histoire du matériau}

La présence d'inclusion joue bien souvent un rôle important sur la durée de vie en fatigue. Le choix du critère de fatigue dépendra donc de la taille de ces inclusions.

\subsection{Influence des inclusions et des contraintes résiduelles}

Pour l'étude de la propagation de fissures, Kitagawa et Takahashi (Kitagawa et al. 1976) utilisent la longueur de fissure $a$ comme paramètre pour prendre en compte la différence de comportement des fissures courtes par rapport aux fissures longues. En effet, la mécanique de la rupture en élasticité linéaire ne peut pas s'appliquer aux fissures courtes dont la condition de plasticité confinée en pointe de fissure n'est pas respectée. Ils représentent le seuil de propagation des fissures longues et la limite d'endurance en fonction de la longueur de fissure. Le même type de diagramme peut être obtenu en substituant la taille des défauts présents dans la pièce à la taille de fissure. On obtient alors deux domaines pour lesquels les comportements en fatigue diffèrent (Figure 4). 


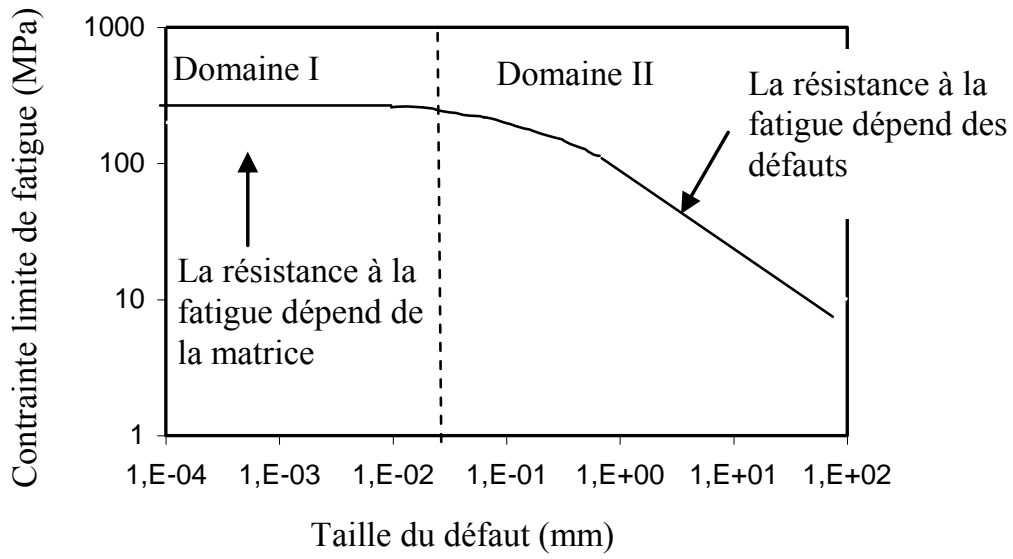

Figure 4 : Influence de la taille des inclusions sur la limite de fatigue

Pour une taille de défaut inférieure à une taille critique (qui dépend de la dureté du matériau), la limite de fatigue ne dépend pas de la taille du défaut. Il est admis que la limite de fatigue est déterminée par la matrice. En revanche, au dessus de la taille critique, la limite de fatigue diminue lorsque la taille de défaut augmente. La limite de fatigue est alors déterminée par les inclusions. En outre, plus la dureté du matériau est grande et plus la taille critique est petite. Dans le domaine I de la figure 4, le modèle de Dang Van est maintenant couramment utilisé en fatigue à grand nombre de cycles. Dans le domaine II par contre, il s'avère nécessaire de tenir compte de la forme, de la taille et de l'orientation des défauts ou inclusions. Le critère de Murakami est alors préféré.

Les contraintes résiduelles présentes dans le matériau ont une influence sur la limite d'endurance. En effet, des contraintes résiduelles de compression améliorent la limite d'endurance du matériau alors que des contraintes de traction diminuent sa limite d'endurance. Pour tenir compte de ces contraintes résiduelles, la solution est de les introduire sous la forme de contraintes statiques. Le premier problème consiste donc à être capable de calculer les contraintes résiduelles à l'issue de la mise en forme. Le deuxième problème est de définir exactement les contraintes résiduelles qui doivent être introduites dans le calcul, car celles-ci peuvent se relaxer durant les cycles de chargement. Toutefois, la relaxation des contraintes résiduelles est un problème très délicat qui sort du cadre de ce travail. 


\subsection{Critère de fatigue pour matériau « sain » : critère de Dang Van}

L'analyse des critères de fatigue pour matériaux dits sains nous a conduit à considérer le critère de Dang Van (Dang Van et al. 1989) qui est un critère de fatigue multiaxial, présentant de bonnes prédictions et particulièrement adapté à la prise en compte de contraintes résiduelles. Le critère se base sur le fait que même si à l'échelle macroscopique la pièce ne subit pas de déformation plastique, à l'échelle microscopique des déformations plastiques sont possibles. Ainsi, le critère consiste à considérer que la rupture ne se fera pas si la réponse des grains les plus défavorablement orientés est adaptée élastiquement. L'utilisation d'une loi de localisation (Lin-Taylor) conduit à utiliser les valeurs du cisaillement microscopique $\tau$ dans un plan critique. Afin de tenir compte du rôle de la pression hydrostatique $\mathrm{P}$ dans le processus de rupture en fatigue, Dang van a introduit ce paramètre dans le critère. Pour qu'il n'y ait pas de rupture en fatigue il faut que le critère de Dang Van soit respecté :

$$
\underset{t}{\operatorname{Max}} \mathbf{I}(t)+a P(t) \underset{\lessgtr}{\exists}
$$

où $a$ et $b$ sont des paramètres matériau. Précisons que le cisaillement microscopique tient compte des phénomènes d'écrouissage isotrope et cinématique. C'est grâce à ce terme qu'il est possible de prendre en compte l'enveloppe de n'importe quel chargement et donc de traiter les cas les plus complexes. Le cisaillement microscopique nécessite l'utilisation d'algorithmes permettant de déterminer le centre de la plus petite hypersphère contenant le trajet de chargement, dans l'espace (de dimension 5) du déviateur des contraintes. Pour plus de précision, le lecteur pourra se référer à (Hopp et al., 1996) et (Ballard et al., 1995).

Les deux constantes $\mathrm{a}$ et $\mathrm{b}$ peuvent être déterminées à partir d'essais de torsion alternée et de flexion alternée.

Une extension anisotrope peut être réalisée de manière phénoménologique, en s'appuyant sur des essais de fatigue réalisés dans plusieurs directions (Ekberg at al., 2001). Il est possible d'introduire trois limites de fatigue différentes suivant trois directions orthogonales, et supposer qu'entre ces directions principales, la limite de fatigue varie suivant un ellipsoïde. Soient les trois directions x, y et z. Alors pour un plan orienté par sa normale $\vec{n}, \vec{n}$ étant défini par les angles $\theta$ et $\varphi$, la limite de fatigue sera donnée par :

$\sigma_{e D V}(\vec{n})=\sigma_{e D V}(\theta, \varphi)=\sqrt{\frac{\sigma_{e x}^{2} \sigma_{e y}^{2} \sigma_{e z}^{2}}{\left(\sigma_{e x} \sigma_{e y} \cos \theta\right)^{2}+\left(\sigma_{e x} \sigma_{e z} \sin \theta \sin \varphi\right)^{2}+\left(\sigma_{e y} \sigma_{e z} \sin \theta \cos \varphi\right)^{2}}}$

où $\sigma_{e D V}(\vec{n})$ est en fait la constante b dans le critère de Dang Van. 


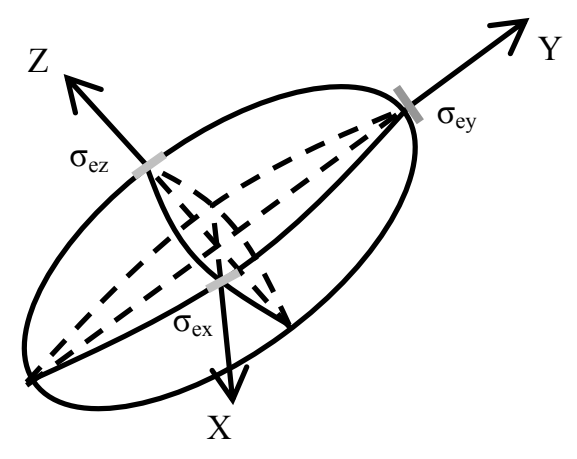

Figure 5:Evolution de la limite de fatigue en fonction de l'orientation du plan de cisaillement

Cette approche semble naturelle du fait que l'on observe effectivement une anisotropie de la limite de fatigue. Ceci étant, cette méthode ne permet pas d'isoler des effets anisotropes dus à des barrières microstructurales et d'autres dus à une texture par exemple. Ainsi, les paramètres du modèle ne sont accessibles qu'au moyen d'essais de fatigue. Pour être employé sur une pièce, dans des zones très déformées et des zones qui le sont moins, cela nécessite de déterminer les paramètres du critère dans trois directions pour plusieurs taux de déformation. Pratiquement, de nombreux essais de fatigue doivent être réalisés (en déterminant par exemple une limite de fatigue avec 10 essais et en considérant 3 taux de déformation, la détermination des paramètres nécessite environ 90 essais de fatigue).

\subsection{Critère de fatigue pour matériau avec défauts : critère de Murakami}

\subsubsection{Caractérisation de la nocivité d'un défaut}

En 1983, Murakami et Endo (Murakami et al. 1983) ont proposé un paramètre géométrique $\sqrt{\text { aire }}$ permettant de caractériser les défauts vis-à-vis de leur nocivité pour la tenue en endurance. Ce paramètre est valable à la fois pour des défauts $2 \mathrm{D}$ et des défauts 3D. $\sqrt{\text { aire }}$ est la racine carrée de l'aire de la projection du défaut sur un plan perpendiculaire à la contrainte principale maximale (Figure 6). 


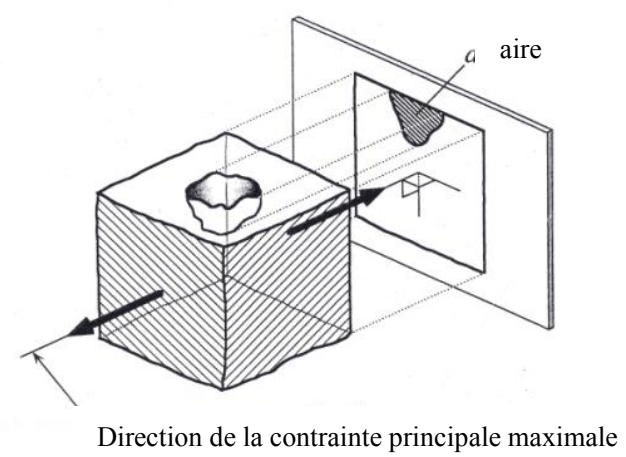

Figure 6: Définition du paramètre aire

A partir de leurs résultats expérimentaux, ils proposent une première formule reliant la limite de fatigue en flexion rotative ou bien en traction compression, en fonction du paramètre $\sqrt{\text { aire }}$. Ce paramètre a ensuite été utilisé dans différents critères, et il s'est montré particulièrement bien adapté pour représenter la nocivité d'un défaut.

\subsubsection{L'équation de Murakami}

Pour prédire la limite de fatigue des matériaux avec défauts, dans le cas de sollicitations uniaxiales, l'équation de Murakami (Murakami et al. 1994) donne de bons résultats. Cette équation permet de déterminer la limite de fatigue $\sigma_{w}$ en fonction de la taille de défaut, lorsque cette taille est supérieure à la taille critique observable sur le diagramme de Kitagawa et Takahashi. Pour les défauts surfaciques :

$\sigma_{w}=1.43 \times \frac{H v+120}{\sqrt{\text { area }}^{1 / 6}}\left(0.5-\frac{R}{2}\right)^{0.226+H v .10^{-4}}$

$H v$ représente la dureté Vickers du matériau, $R$ est le rapport des contraintes :

$R=\frac{\sigma_{\min }}{\sigma_{\max }}$

Cette équation (5) a été établie en assimilant le défaut à une fissure pour laquelle l'amplitude du facteur d'intensité de contraintes est proportionnelle au paramètre area à la puissance 0,25 (en flexion rotative ou en traction compression). Le seuil de 
propagation a été déterminé expérimentalement et il dépend également du paramètre area. L'équation de Murakami est issue de ces deux relations. Elle a été établie pour des contraintes uniaxiales présentant des rapports de charge compris entre 0 et -3 . Son domaine de validité est donc réduit à ce type de chargement.

\subsubsection{Extension multiaxiale de l'équation de Murakami}

Des travaux ont alors été menés afin d'étendre la gamme de chargement à des contraintes biaxiales (Beretta et al. 2000). Partant du principe que la limite de fatigue est directement liée à la propagation des fissures en mode I, Beretta et Murakami estiment que le rapport des limites de fatigue en flexion et en torsion est égale à :

$$
\frac{\tau_{w}}{\sigma_{w}}=\frac{K_{I, \text { tens }}}{K_{I, \text { shear }}}
$$

Ce rapport, d'après les données de la littérature, est égal à 0,85 . Ainsi, pour retrouver ce résultat, ils effectuent des calculs en 3 dimensions, pour des fissures émanant de trous sphériques ou ellipsoïdaux, soumises à des contraintes biaxiales. Cela leur permet d'obtenir une fonction décrivant de manière plus générale l'évolution du rapport $K_{I, \text { tens }} / K_{I, \text { biaxial }}$, c'est-à-dire le rapport des facteurs d'intensité de contraintes pour une contrainte uniaxiale et des contraintes biaxiales.

$$
\frac{K_{I, \text { tens }}}{K_{I, \text { shear }}}=1+0,1804 \cdot \frac{\sigma_{1}}{\sigma_{2}}+0,0329 \cdot\left(\frac{\sigma_{1}}{\sigma_{2}}\right)^{2}
$$

Ainsi, la limite de fatigue pour n'importe quelle combinaison de chargements en phase de traction et de torsion, peut être calculée en utilisant cette équation.

Dans la suite, l'équation de Murakami est étendue aux configurations multiaxiales, dans le cas de contraintes quelconques. La question importante est de savoir comment étendre le paramètre R (rapport des contraintes) lorsque celui-ci n'a plus de sens?

Les réflexions menées nous ont conduit à introduire la notion de contraintes effectives $\left(\sigma_{w}-\sigma_{t h}\right)$ (Caillet et al. 2005), déterminées par la différence entre contraintes réelles et une contrainte $\sigma_{\text {th }}$ dite contrainte seuil. Cette approche s'inspire de la mécanique linéaire de la rupture dans laquelle on trouve la notion de facteur d'intensité de contraintes seuil au dessous duquel la fissure n'est pas ouverte. L'amplitude du facteur d'intensité de contraintes qui doit être considérée pour le calcul de la propagation de la fissure est alors ramenée à l'amplitude des facteurs d'intensités de contraintes supérieurs à la valeur seuil. Bien que l'on ne transpose pas 
directement les mécanismes, la contrainte seuil traduit ici la même idée, à savoir qu'en dessous de cette contrainte seuil, la contribution à la fissuration en fatigue est nulle.

Nous nous ramenons à des directions principales de contraintes, et pour chacune de ces directions nous calculons des contraintes seuil $\left(\sigma_{t h}^{I}\right.$ et $\sigma_{t h}^{I I}$ sur la

Figure 7) grâce à l'équation uniaxiale de Murakami.

L'équation de Murakami peut alors être réécrite :

$\sigma_{w}-\sigma_{t h}=1.43 \times \frac{H v+120}{\sqrt{\text { area }}^{1 / 6}}$

En combinant la fonction de poids à cette approche faisant intervenir la contrainte seuil, nous disposons d'un critère d'endurance illimité multiaxial pour des matériaux avec défauts. Ce critère est capable de prendre en compte l'effet de contraintes résiduelles, et il ne nécessite pas d'essais expérimentaux.

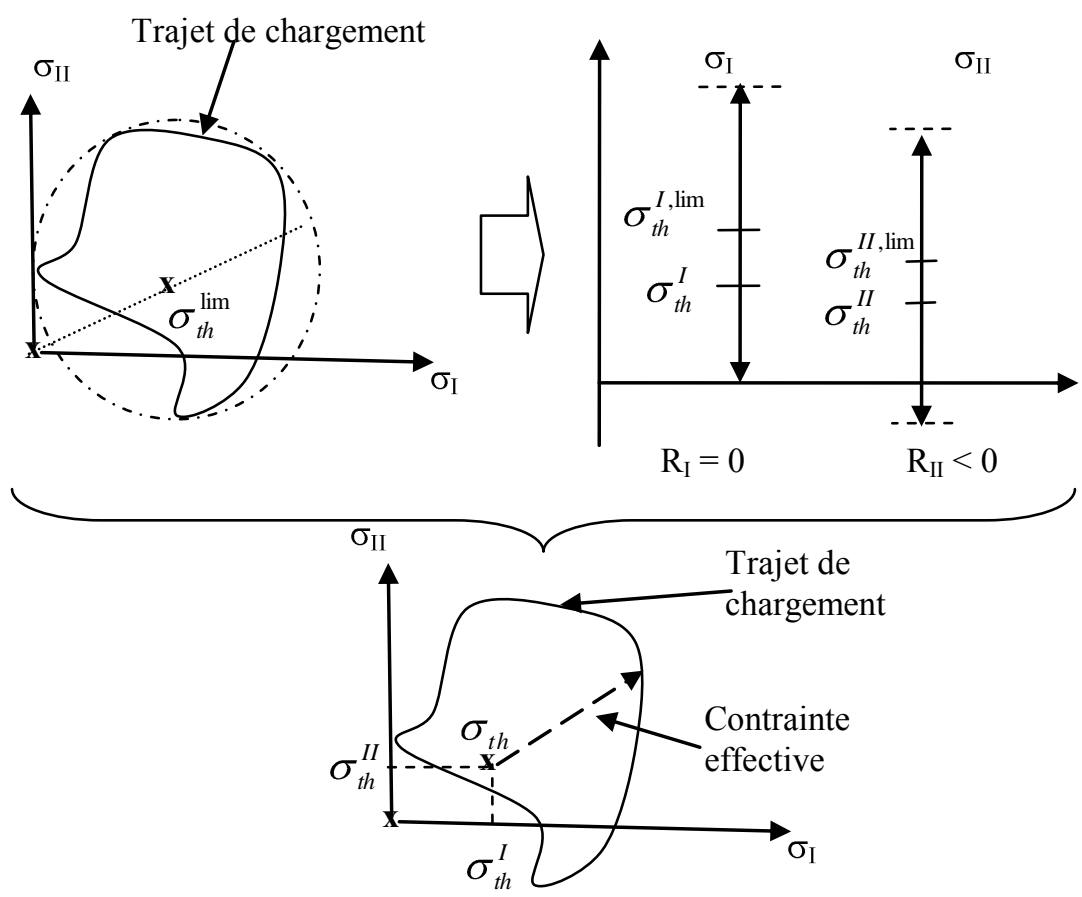


Figure 7: Exemple de détermination des contraintes effectives pour un chargement biaxial

\subsubsection{Critère de Murakami et anisotropie}

L'équation initiale de Murakami intègre le paramètre aire, défini précédemment. En présence d'inclusions allongées, ce paramètre est amené à varier en fonction de la direction de l'allongement.

Lors de la déformation de la matière, les inclusions malléables se déforment elles aussi. Une inclusion non déformée sera représentée par une sphère tandis qu'une inclusion déformée sera représentée par un ellipsoïde de révolution. Pour calculer le paramètre aire, il faut alors prendre l'aire de l'ellipse résultant de la projection de l'ellipsoïde de révolution suivant la direction de la contrainte principale. La Figure 8 montre qu'en fonction de la direction de l'allongement de l'inclusion et de la direction des contraintes, le paramètre aire change de valeur.

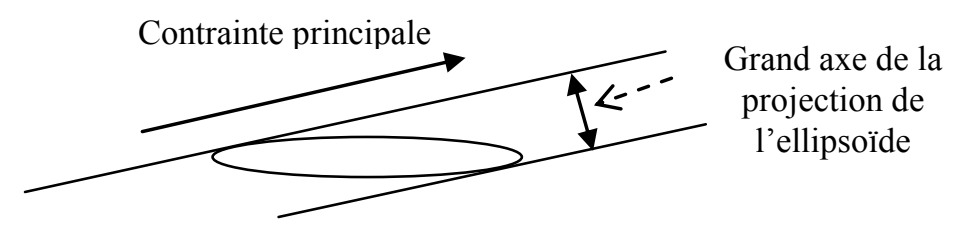

Figure 8: Calcul du paramètre aire pour les inclusions de forme ellipsoïdale

Par exemple, pour un essai de traction-compression, la valeur du paramètre aire est minimale lorsque la direction de l'allongement de l'inclusion est identique à la direction des sollicitations, ce qui correspond à une éprouvette prise dans le sens long. C'est cette configuration qui résistera donc le mieux à la fatigue, ce qui correspond effectivement aux observations expérimentales. Le critère de Murakami intègre donc intrinsèquement l'anisotropie engendrée par l'élongation des inclusions. Pour établir le lien avec la mise en forme, il faut que le vecteur fibrage soit une indication de la forme et la direction des inclusions au sein de la pièce.

Le critère de Murakami et son extension sont présentés plus en détail et validés dans (Caillet et al. 2005). 


\section{Application à un poussoir de soupape}

\section{- Mise en forme et chargement en fatigue}

La pièce servant pour le cas test industriel est un poussoir qui sert d'interface lors de la transmission du mouvement entre l'arbre à cames et la soupape (Figure 9).
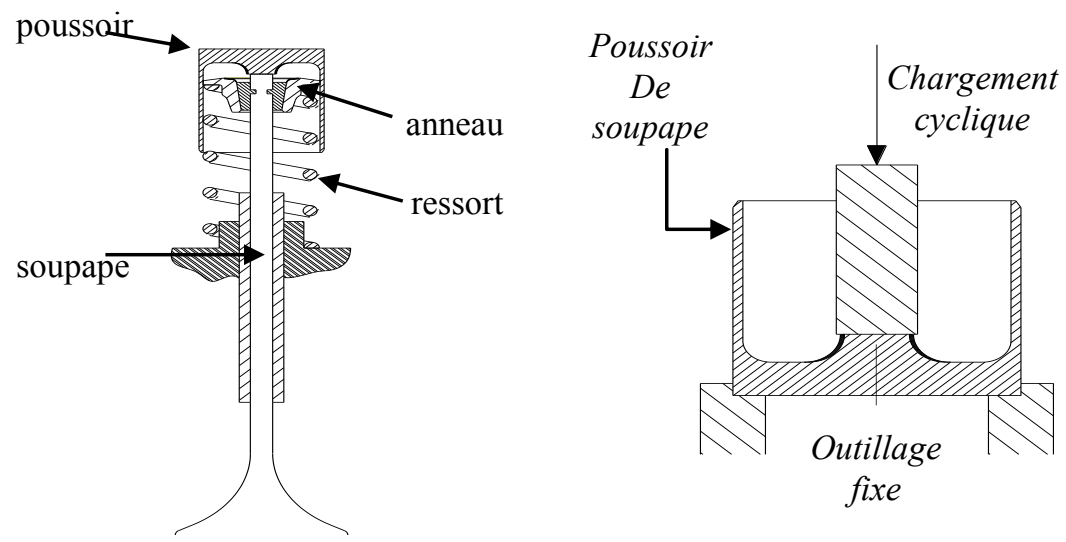

Figure 9: Fonctionnement du poussoir de soupape et chargement simplifié

Pour des raisons de confidentialité, la gamme de mise en forme n'est pas détaillée ici. La figure 10 montre la géométrie de la pièce obtenue à la fin des différentes étapes de mise enforme. Chacune des étapes étant modélisées par FORGE3 ${ }^{\circledR}$, nous prenons en compte l'histoire du matériau: déformations plastiques, contraintes résiduelles, fibrage.

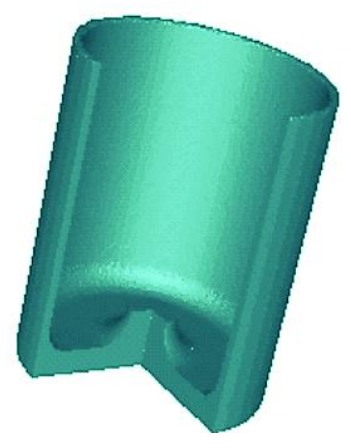


Figure 10: Géométrie finale du poussoir obtenu par simulation numérique des différentes étapes de mise en forme

Un fibrage homogène est introduit initialement afin de reproduire le fibrage du barreau de départ. Le vecteur fibrage est ensuite calculé à chaque étape. Le poussoir de soupape obtenu intègre donc un fibrage final ainsi que des contraintes résiduelles (calculées par Forge $3 \AA$ ). Un calcul de fatigue est alors réalisé sur cette pièce, en appliquant un chargement cyclique simplifié décrit en figure 9. Ce chargement est introduit de façon artificielle et est volontairement exagéré de façon à provoquer l'apparition de fissures de fatigue. Ce chargement est superposé aux contraintes résiduelles (nous noterons qu'aucun calcul de relaxation des contraintes résiduelles n'est réalisé).

La Figure 11.a montre les valeurs du critère de Murakami obtenues (pour des tailles d'inclusions de l'ordre de 20 microns si l'inclusion est sphérique) et pour les même sollicitations que celles appliquées expérimentalement.

Deux zones critiques apparaissent. La première au niveau des rayons de congé de l'ergot central, et la seconde sur le côté inférieur du poussoir, à un rayon $r$ du centre. La figure 11.b montre la présence de fissures localisées à ces mêmes endroits pour les pièces testées en fatigue expérimentalement.

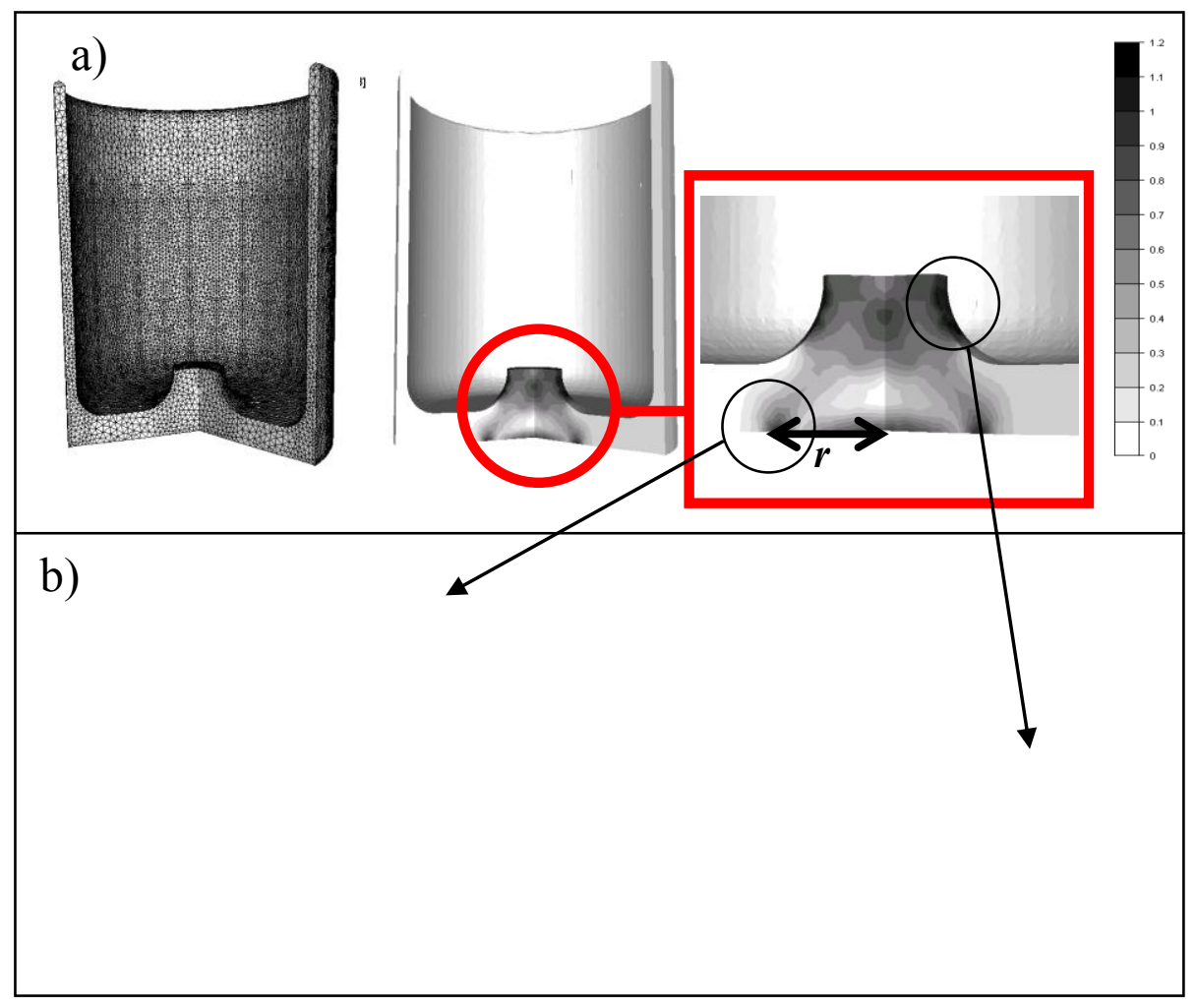



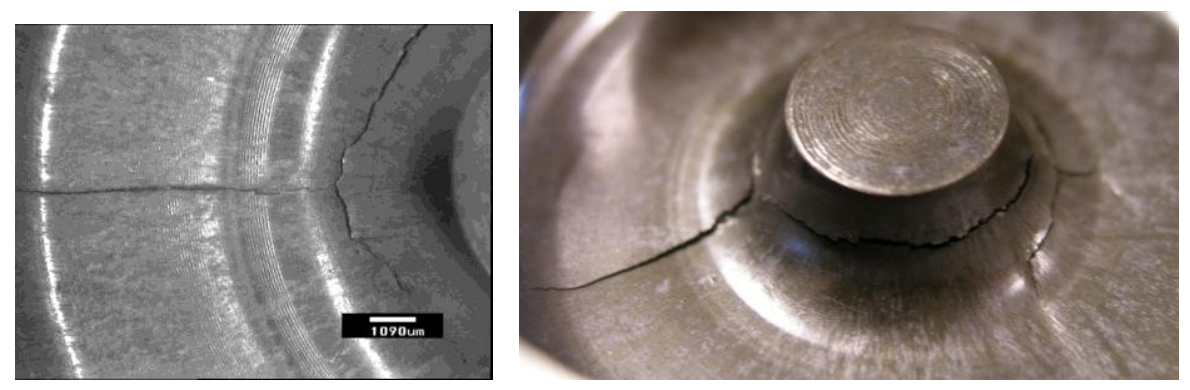

Figure 11: a) Maillage de la géométrie finale du poussoir et champ des valeurs $d u$ critère de Murakami - b) fissures observées expérimentalement

\section{- Vers l'optimisation du poussoir de soupape}

Des outils d'optimisation sont en cours de développement de façon à améliorer la tenue mécanique de pièces soumises à des chargements cycliques. Les paramètres d'optimisation peuvent être soit l'orientation du fibrage initial, soit la géométrie du poussoir de soupape. Des algorithmes basés sur les stratégies d'évolution sont développés en parallèle dans le cadre de l'étude de Thien Tho Do (Fourment et al. 2005). Ces algorithmes peuvent être utilisés au travers d'une boucle d'optimisation intégrant les phases de forgeage et de calcul de fatigue (figure 12).

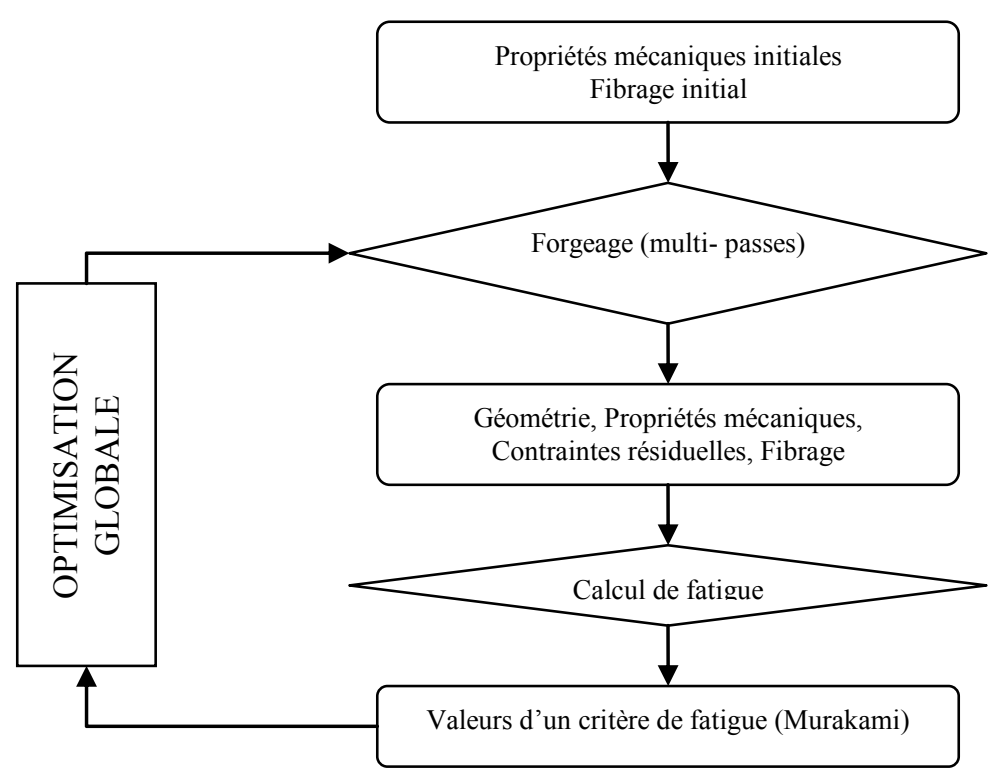


Figure 12: Boucle d'optimisation

Dans la suite, l'influence de deux paramètres sur les résultats fournis par le critère de Murakami ont été testés :

- la hauteur de l'ergot central du poussoir de soupape : aucune influence significative n'a été observée sur les valeurs du critère de Murakami en modifiant cette hauteur jusqu'à $10 \%$ de sa valeur initiale.

- L'orientation du fibrage initial : la figure 12 montre la répartition des valeurs du critère de Murakami dans 3 configurations différentes : a) sans fibrage initial, b) avec un fibrage initial suivant la direction $\mathrm{z}, \mathrm{c}$ ) avec un fibrage initial suivant la direction y.

La figure 13 montre que les valeurs de Murakami sont significativement différentes suivant que l'on considère le fibrage ou non. Nous observons des valeurs importantes du critère dans 3 zones distinctes (zone $\mathrm{A}, \mathrm{B}$ et $\mathrm{C}$ de la figure 13. La valeur maximale est obtenue dans la zone A.

Le critère de Murakami montre bien que l'on obtient des valeurs plus faibles lorsque l'on intègre un fibrage initial orienté suivant $z$. Inversement, si le fibrage initial est orienté suivant y, la valeur du critère de Murakami est maximale. Nous voyons donc que la bonne maîtrise du fibrage permet d'obtenir une meilleure résistance en fatigue. Notons que la meilleure configuration correspond au procédé réel.

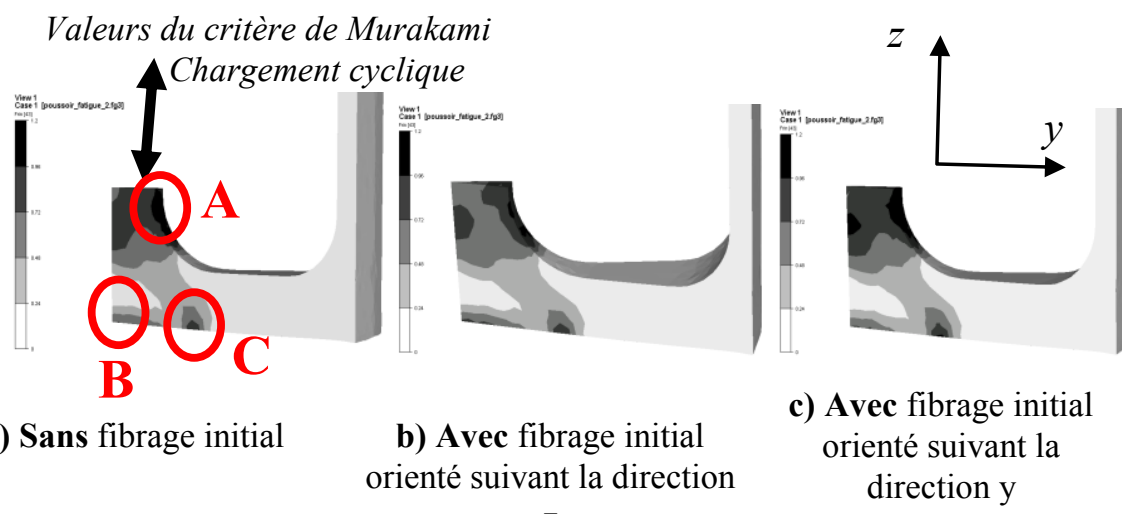

Figure 13: Valeurs du critère de Murakami en fonction du fibrage initial 


\section{Conclusion}

L'objectif général de cette étude était de mettre en place une chaîne de simulation complète, intégrant à la fois l'étape de mise en forme et de dimensionnement. Pour cela, une première étude sur la modélisation du forgeage intégrant une variable décrivant le fibrage a été menée. Deux modèles de fatigue à grand nombre de cycles ont ensuite été développés : le modèle de Dang Van et le modèle de Murakami. Ce dernier a été étendu pour être appliqué en conditions multiaxiales, plus proches des conditions industrielles.

Un poussoir de soupape a ensuite servi de cas d'étude. Les 4 passes de forgeage du poussoir de soupape ont été modélisées. La géométrie, les contraintes résiduelles ainsi que le fibrage ont ensuite servi de données d'entrée pour l'étape de dimensionnement. Le critère de Murakami a localisé deux zones probables de rupture qui ont également été observées expérimentalement.

Le module d'optimisation développé dans une étude annexe (Fourment et al. 2005) a ensuite été connecté à cette chaîne de simulations virtuelle. Cela a permis de mettre en évidence l'influence de l'orientation du fibrage initial du lopin sur les valeurs du critère de Murakami.

Cette méthodologie demande maintenant à être fiabilisée et étendue. Plusieurs pistes peuvent être envisagées: enrichir le modèle direct en intégrant des informations mécaniques et microstructurales plus fines (endommagement, taille de grains, état inclusionnaire, ...), améliorer le module d'optimisation de façon à pouvoir modifier la forme des outils pour les différentes passes de forgeage, ou encore intégrer de nouveaux procédés dans la chaîne de simulation directe, tels que la découpe ou encore le traitement thermique.

Ce travail a été réalisé dans le cadre du projet OPTIMAT, financé par le Réseau National Technologies et Logiciels (RNTL). Les auteurs souhaitent remercier également la société AMIS pour le travail réalisé sur le poussoir de soupape.

\section{Références}

Ballard P., Dang Van K., Deperrois A., Papadopoulos Y.V., «High cycle fatigue and a finite element analysis », Fatigue Fract. Engng. Mater. Struct. Vol 18, No. 3, 1995, p. 397-411.

Beretta, S. and Murakami Y., "SIF and threshold for small cracks at small notches under torsion", Fatigue Fract. Engng. Mater. Struct., 23, 2000, p. 97-104.

Bouchard P.O., Fayolle, S. and Mocellin K. "3D Numerical Modeling of Mechanical Joining Processes - From Joining down to Structural Analysis", The Eighth International Conference on Computational Structures Technology, Las Palmas de Gran Canaria, Spain, 12-15 September 2006. 
Caillet, N., Bouchard, P.-O., Chastel, Y., "Multiaxial Approach of Murakami Equation and Applications to Forged Parts", 4th International Conference on Fracture and Damage Mechanics, Mallorca, Spain, July 12-14, 2005.

Coupez, T., Digonnet, H. and Ducloux, R., "Parallel meshing and remeshing", Applied Mathematical Modelling, vol. 25, 2000, p. 153-175.

Dang Van, K. , Griveau, B. and Message, O., "On a new multiaxial fatigue limit criterion: Theory and application", Biaxial and Multiaxial Fatigue, EGF 3, Mechanical Engineering Publications, London, 1989, p. 479-496.

Ekberg, A., Sotkovszki, P., Anisotropy and rolling contact fatigue of raiway wheels, International Journal of Fatigue, 23, 2001, p. 29-43.

Fourment, L., Do, T.T., Habbal, A. and Bouzaiane, M., "Gradient, non-gradient and hybrid algorithms for optimizing 2D and 3D forging sequences", $8^{\text {th }}$ International ESAFORM Conference on Material Forming, Cluj-Napoca, Romania, 2005.

Hopp, H. and Reeve P., "An algorithm for computing the minimum covering sphere in any dimension”, http://www.mel.nist.gov/msidlibrary/doc/hopp95.pdf, 1996.

Kitagawa H. and Takahashi S., "Applicability of fracture mechanics to very small cracks or the cracks in the early stage", Proceedings $2^{\text {nd }}$ Int. Conference Mech. Behaviour Mater. ICM2, Boston, 1976, p.627-631.

Murakami, Y., Endo, M., "Effects of defects, inclusions and inhomogeneities on fatigue strength", Fatigue, Vol 16, 1994, p. 163-182.

Murakami, Y., Endo, M., "Quantitative evaluation of fatigue strength of metals containing various small defects or cracks", Engineering Fracture Mechanics, Vol. 17, No. 1, 1983, p. $1-15$. 